\title{
Intraoperative Radiation Therapy and Chemotherapy for Huge Choroid Plexus Carcinoma in an Infant - Case Report-
}

\author{
Toshihiro KumABE, Teiji TOMINAGA, Takeo Kondo, \\ Takashi YOSHIMOTO, and Takamasa KAYAMA* \\ Department of Neurosurgery, Tohoku University School of Medicine, Sendai; \\ ${ }^{*}$ Department of Neurosurgery, Yamagata University School of Medicine, Yamagata
}

\begin{abstract}
A 2-month-old boy presented with a huge choroid plexus carcinoma in the left cerebral hemisphere. Almost total resection of the tumor was achieved, but a remnant abruptly enlarged 5 months later, requiring reoperation. After radical resection of the tumor, intraoperative radiation (10 Gy) was delivered. Postoperative chemotherapy was performed with methotrexate and vincristine, selected on the basis of in vitro chemosensitivity testing. No recurrence of the tumor was detected 18 months after the second operation. Radical surgery with these adjuvant therapies may be a good method to control this aggressive disease in infants.
\end{abstract}

Key words: chemotherapy, chemosensitivity test, choroid plexus carcinoma, intraoperative radiation therapy, magnetic resonance imaging, operation

\section{Introduction}

Neoplasms derived from the epithelium of the choroid plexus are rare, forming about $0.4-0.6 \%$ of all intracranial tumors. ${ }^{25)}$ They are classified into the benign papilloma type and malignant carcinoma type. ${ }^{12,19,25)}$ Choroid plexus carcinoma is rarer and comprises $10-20 \%$ of choroid plexus tumors. ${ }^{9,15,17)}$ The prognosis for this disease is generally poor. ${ }^{5,7,10,17)}$ Radical surgical excision and postoperative irradiation are the best treatments for prolonged survival in patients with choroid plexus carcinoma. ${ }^{6}$ However, radiotherapy in young patients is controversial because neurological impairment may subsequently occur. ${ }^{11)}$ The benefits of adjuvant chemotherapy are still under discussion. ${ }^{17}$

We describe a case of large choroid plexus carcinoma managed by radical resection, intraoperative radiation therapy (IORT), ${ }^{14,20,21)}$ and chemotherapy using agents selected by in vitro chemosensitivity testing. ${ }^{1,3)}$

Received June 15, 1995; 1995

\section{Case Report}

A 2-month-old boy was admitted to our hospital because of head enlargement. His head circumference was $33 \mathrm{~cm}$ at birth, and reached $48 \mathrm{~cm}$ at 2 months old when he became progressively more irritable. On admission, his body weight was $6700 \mathrm{~g}$ and his head circumference was $48.5 \mathrm{~cm}$. The left side of his cranium was tender and projected unevenly. The sun-set phenomenon was observed. The hypertonic lower extremities showed sustained clonus at the knees and ankles. Plain craniography showed enlargement of the skull and marked decalcification of the left side of the vertex. Magnetic resonance (MR) imaging showed a large ventricular tumor which was about 9 $\mathrm{cm}$ in diameter. The tumor was composed of an intensely enhanced solid portion, signal void foci, and multiple cysts (Fig. 1). Angiography demonstrated a prominent tumor stain fed by the anterior and posterior choroidal arteries, the cortical branches of the middle cerebral artery, and the anterior cerebral artery. Early draining veins were seen within the tumor stain.

He underwent a left frontotemporoparietal craniotomy in August 1993. The atrophic cerebral 


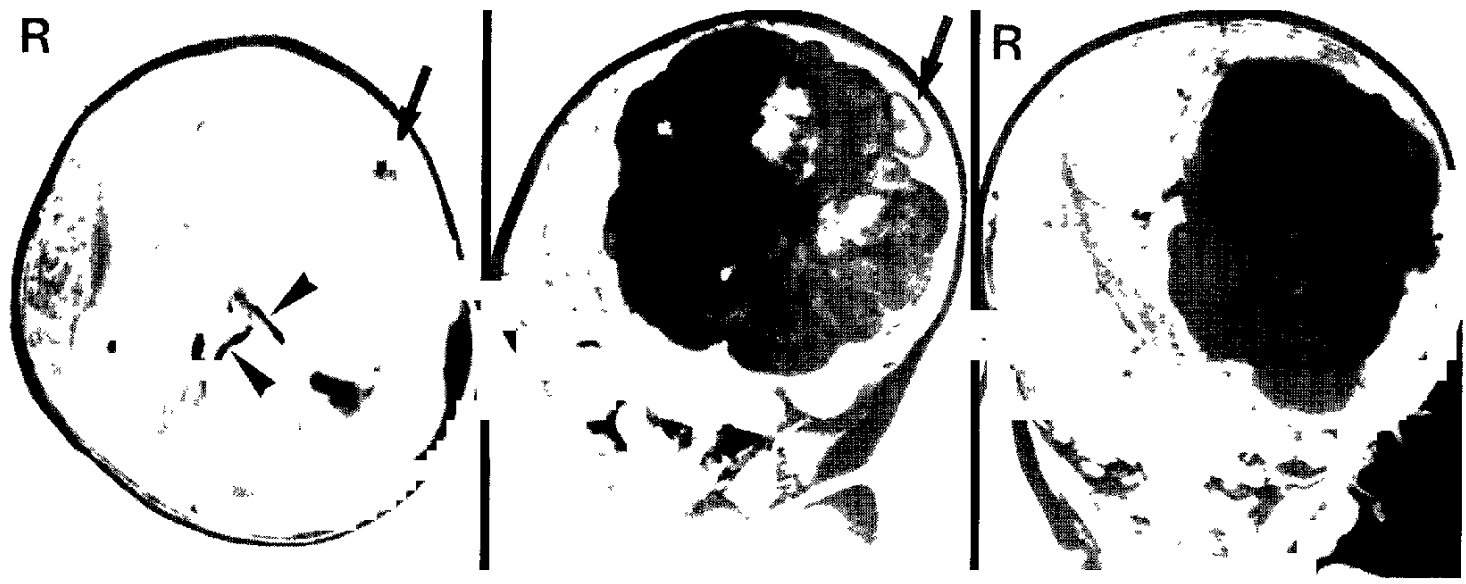

Fig. 1 Preoperative MR images with gadolinium enhancement, axial (left), sagittal (center), and coronal (right) views, demonstrating the huge enhanced tumor in the left hemisphere, the presence of high flow large vessels (arrowheads), and multiple cyst formations around the tumor (arrows).

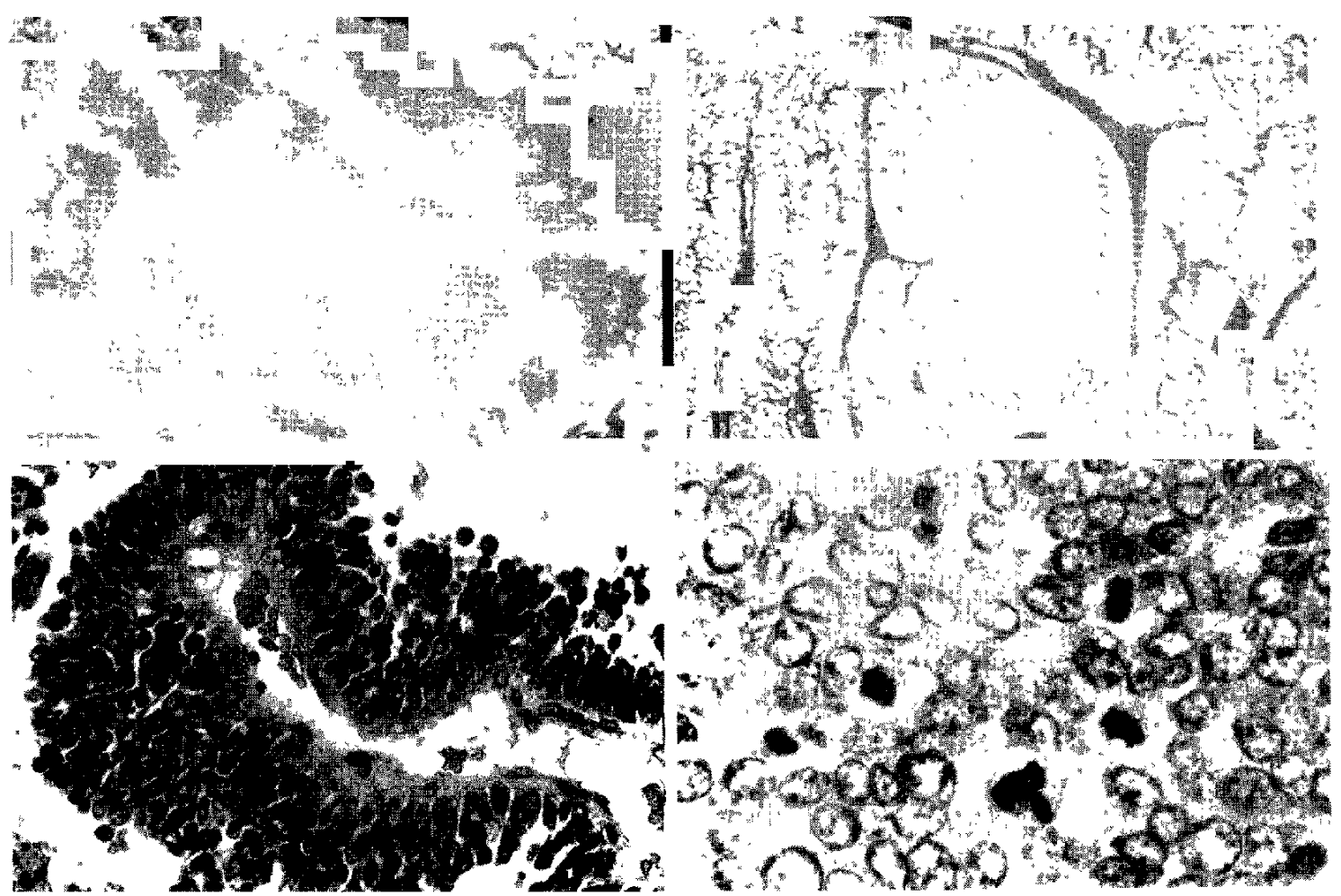

Fig. 2 Photomicrographs of the first tumor specimen. upper left: The neoplastic cells have a uniformly columnar shape and papillary arrangement. HE stain, $\times 200$. upper right: The neoplastic cells show solid growth patterns. HE stain, $\times 100$. lower left: The cells vary considerably in size, with marked variation in nucleus size, shape, and staining intensity. HE stain, $\times 200$. lower right: Many mitoses are present. HE stain, $\times 400$.

cortex overlying the tumor was incised. Initially the tumor blood supply from the choroid plexus could not be secured. We debulked the tumor internally and separated the surrounding gliosis tissue via the 


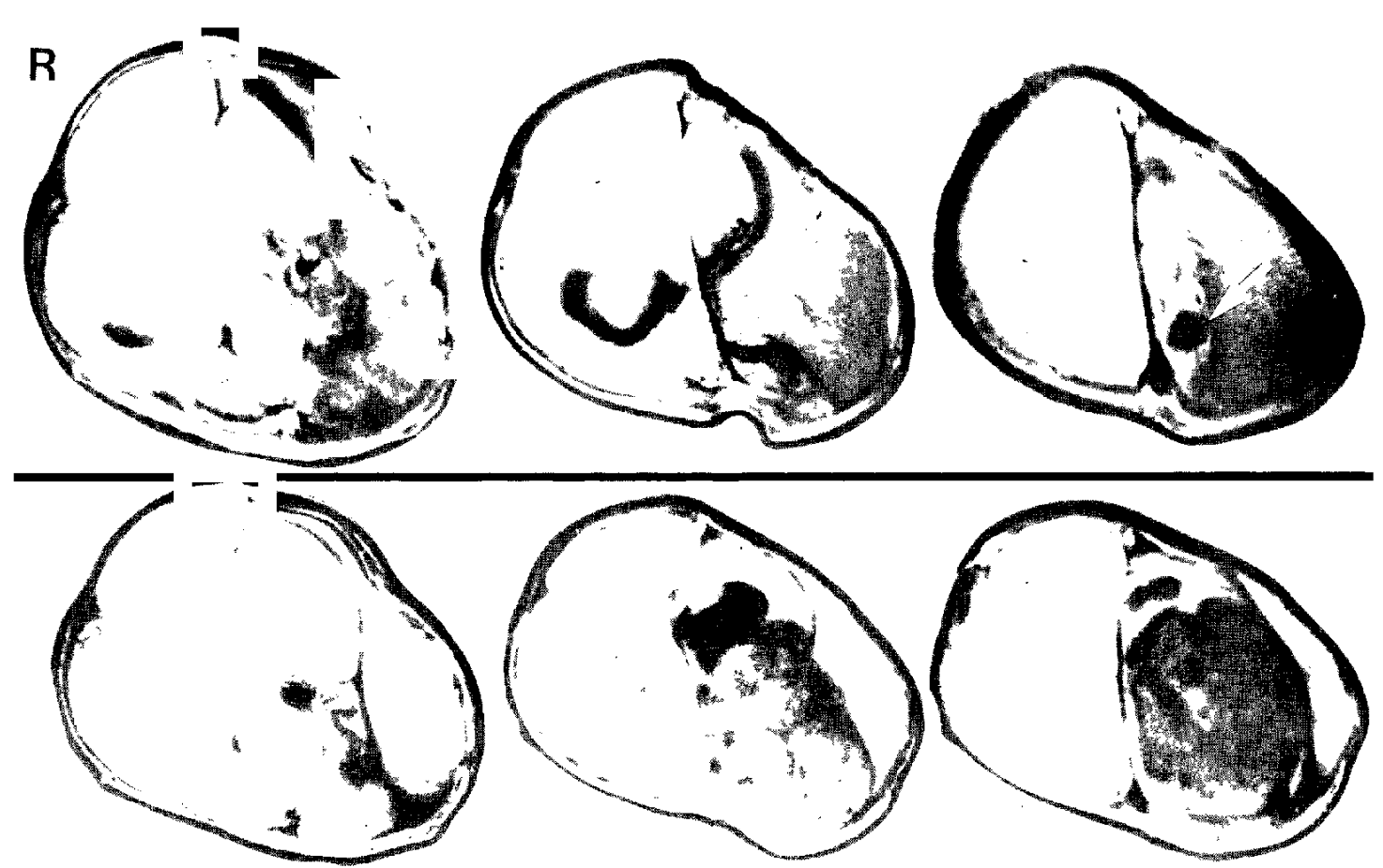

Fig. 3 Axial MR images with gadolinium enhancement, 2 months after the first operation (upper row) showing the almost total resection of the huge tumor, with clots in the ventricles and the subdural space, and a small enhanced mass in the parietal region (arrow); and before the second radical operation (lower row) showing the enhanced mass at the parietal region has enlarged abruptly.

multiple peritumoral cyst formations. These procedures were accompanied by substantial hemorrhage. Finally, the very vascular-rich tumor was totally resected en bloc. He lost as much as $3805 \mathrm{ml}$ of blood, but his intraoperative and postoperative course was uneventful.

Histological examination of the tumor specimen revealed choroid plexus carcinoma. The tumor included normal-appearing choroid plexus, papillomatous proliferation, and carcinomatous lesions (Fig. 2). The tumor cells in the papillomatous areas had a uniform columnar shape and papillary arrangement. The carcinomatous areas contained tubular and solid growth patterns. The nuclei showed mitoses and marked variation in size, shape, and staining intensity. There were extensive areas of necrosis and hemorrhage. Proliferation of vascular structures was also observed.

Postoperative computed tomography revealed total removal of the tumor. A ventricular shunt was implaced 3 weeks after the first operation due to hydrocephalus. Cytological examination showed his cerebrospinal fluid contained no malignant cells. Postoperative MR imaging 2 months after the first operation showed almost total resection of the huge tumor except for a small enhanced mass in the parietal region (Fig. 3 upper row). Hypertonicity of the bilateral lower extremities was diminished. His weight increased to $7200 \mathrm{~g}$ after discharge home. $\mathrm{He}$ was followed up with monthly MR imaging, which showed abrupt enlargement of the enhanced mass at the parietal region (Fig. 3 lower row). He was readmitted to our hospital 5 months after the first operation. At this time, right motor weakness was observed.

A second left frontotemporoparietal craniotomy was performed in February 1994. The tumor was covered with an internal capsule of chronic subdural hematoma. The tumor was dissected from the tentorial surface, the falx, and the frontal lobe, and then totally resected en bloc (Fig. 4 upper row). IORT was performed using $10 \mathrm{~Gy}$ of beta-irradiation. The irradiation was designed to penetrate 1$1.5 \mathrm{~cm}$ deeper than the surface of tumor cavity. The radiation field was $4 \mathrm{~cm}$ in diameter and focused on the choroid plexus. The histology of the specimens was the same as those from the first operation.

The surgical specimen was mechanically and enzymatically dissociated under aseptic conditions. The digested tumor tissue was passed through a $70 \mu \mathrm{m}$ 

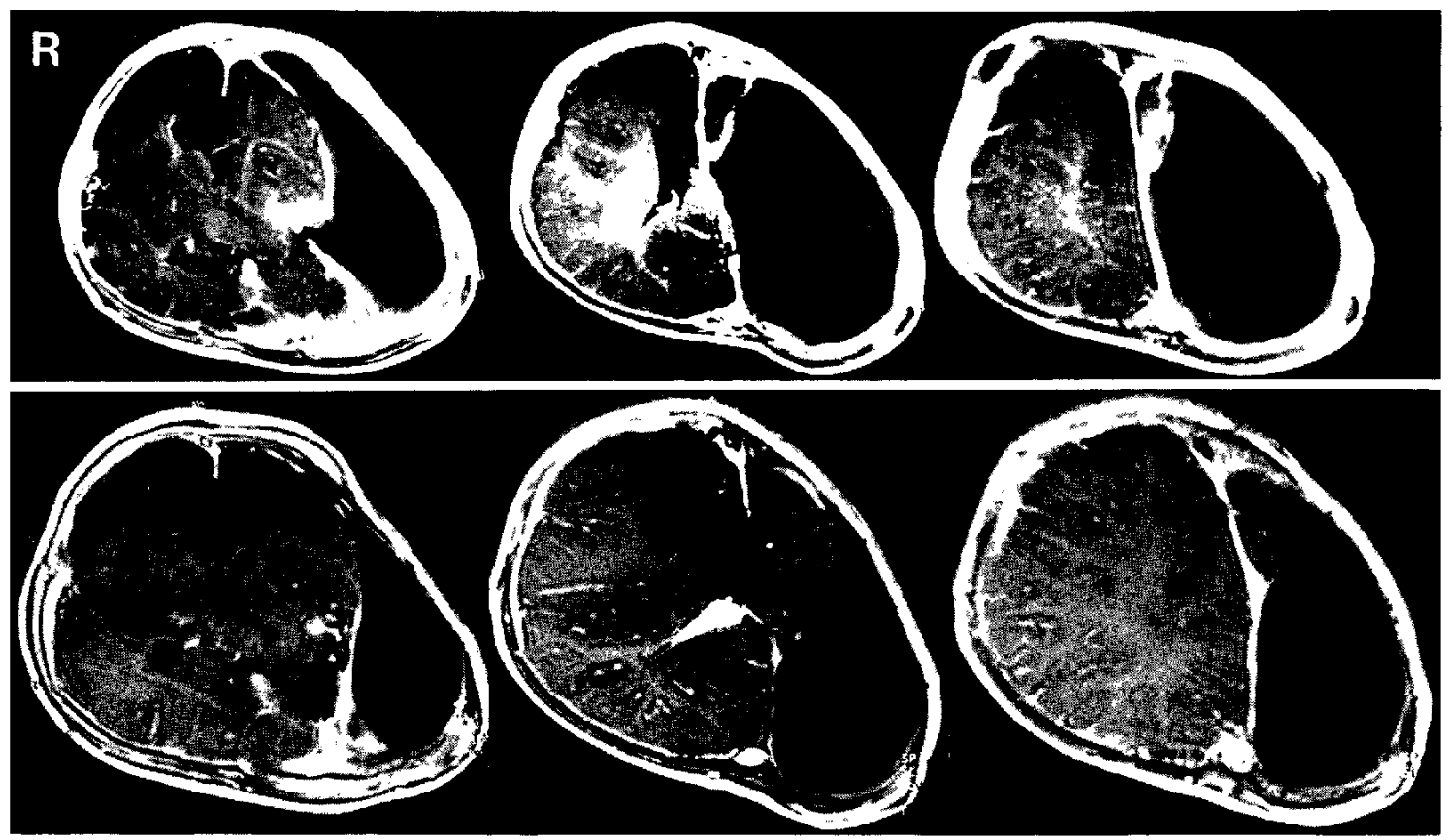

Fig. 4 Axial MR images with gadolinium enhancement, 2 weeks after the second operation (upper row) showing the total removal of the tumor; and 18 months after the second operation (lower row) showing no tumor recurrence.

pore cell strainer and incubated on culture dishes in Dulbecco's minimum essential medium containing $20 \%$ fetal calf serum under conventional culture conditions. Cisplatin, etoposide, 1-(4-amino-2-methyl-5pyrimidinyl)methyl-3-(2-chloroethyl)-3-nitrosourea hydrochloride ( $\mathrm{ACNU}$ ), vincristine, and methotrexate were added to these primary cultures. Growth inhibition was evaluated with a microculture tetrazolium assay. ${ }^{3)}$ Culture plates were tested in duplicate. Vincristine and methotrexate were the most effective growth inhibitors. ACNU and cisplatin achieved minimal growth inhibition even at high concentrations. The concentration achieving 90\% growth inhibition $\left(\mathrm{IC}_{90}\right)^{1)}$ was $0.9 \mathrm{ng} / \mathrm{ml}$ for vincristine and $38 \mathrm{ng} / \mathrm{ml}$ for methotrexate.

Based on the chemosensitivity testing, $0.5 \mathrm{mg}$ of vincristine and $2 \mathrm{mg}$ of methotrexate were administered intravenously per day. The expected serum concentration to be obtained by intravenous injection was higher than the $\mathrm{IC}_{90}$ shown by in vitro chemosensitivity testing. ${ }^{16,26)}$ The blood-brain barrier is absent at the choroid plexus, so these agents should easily penetrate into the tumor tissue. This protocol was performed once a week, for a total of four times.

He was discharged 2 months after the second operation. His right motor weakness, observed before the second admission, gradually improved. His body weight increased to $9500 \mathrm{~g}$. He received maintenance therapy of intravenous administration of $0.5 \mathrm{mg}$ of vincristine and $2 \mathrm{mg}$ of methotrexate once a month. MR imaging 18 months after the second radical operation showed no recurrence of the tumor (Fig. 4 lower row). His development was evaluated as that of a 7-month-old boy.

\section{Discussion}

There are several histological criteria for the diagnosis of choroid plexus carcinoma. Many authors ${ }^{12,19,24)}$ suggest tumor invasion into brain tissue as a diagnostic criterion. However, tumor invasion is difficult to demonstrate if the surgical specimen does not contain surrounding brain tissue. Ellenbogen et al. ${ }^{97}$ suggest the following are essential for the diagnosis of choroid plexus carcinoma: areas with loss of differentiated papillary architecture, and malignant cellular features such as mitoses and nuclear pleomorphism. Our case fulfilled all these criteria.

The biological behavior of choroid plexus carcinoma is poorly characterized. ${ }^{17)}$ The histological appearance of choroid plexus carcinoma does not necessarily indicate the biological behavior, as many highly anaplastic choroid plexus neoplasms are clinically be- 
nign. ${ }^{4)}$ However, the survival period for patients with choroid plexus carcinoma is generally poor. ${ }^{5,7,17)}$ The time from onset of symptoms to death was approximately 9 months in children and 3.5 years in adults. ${ }^{\text {? }}$ More recently, Ellenbogen et al. ${ }^{\text {9) }}$ reported a 5-year survival rate of $50 \%$ in 14 children with choroid plexus carcinoma.

Surgery may have a critical impact on the progression-free survival of the patient with choroid plexus carcinoma. Operative morbidity and mortality of choroid plexus tumors in children are associated mainly with excessive blood loss. ${ }^{9)}$ Our patient had an uneventful intraoperative and postoperative course despite the loss of as much as $3805 \mathrm{ml}$ of blood, which was due to modern neuroanesthetic techniques. Surgical success with this disease absolutely requires the constant attention of well-trained pediatric anesthesiologists. ${ }^{23)}$ In addition, the presence of the peritumoral cyst formations ${ }^{13,22)}$ contributed to the successful dissection and resection of this very vascular-rich tumor.

Radiation therapy is also necessary at least at recurrence. ${ }^{15,17)}$ In fact, most children with choroid plexus carcinomas achieving long-term survival had received radiation therapy. ${ }^{10)}$ However, extensive radiation of the young causes severe intellectual and endocrinological sequelae. ${ }^{11)}$ In our patient, we found that surgical resection alone was not enough to control this aggressive tumor because of the very rapid growth of the recurrent tumor. Therefore, IORT and chemotherapy with agents selected by chemosensitivity test were used to control the tumor and minimize neurological damage. The efficacy of IORT remains to be established. IORT is reported to be significantly effective in the "induction of remission" following surgical excision of malignant gliomas. ${ }^{14,20,21)}$ IORT minimizes damage to normal tissue through the direct visualization of the tumors during surgical exploration. This advantage is particularly beneficial to young patients. The development of our patient was delayed significantly. However, this may have been the result of primary brain damage by tumor growth rather than radiation therapy.

Only a few cases of choroid plexus carcinoma have been treated successfully by chemotherapy alone or in combination with radiation therapy..$^{1,2,8,10)}$ Eightdrugs-in-one-day treatment ${ }^{18)}$ was reported effective with this disease. ${ }^{10)}$ However, the toxicity of chemotherapy cannot be ignored. ${ }^{18)}$ Effective agents against the tumor in vitro are not always effective in vivo, but agents ineffective in vitro must be ineffective in vivo. Chemosensitivity testing showed administration of ACNU or cisplatin would be ineffective in our patient because these agents did not in- hibit the tumor growth in vitro. Such in vitro testing may be useful to obtain optimal effect and minimal toxicity by chemotherapy.

The optimum treatment for choroid plexus carcinomas is still unclear. We think a better prognosis for choroid plexus carcinoma can be obtained by total resection of the tumor with suitable adjuvant therapy, such as IORT and chemotherapy using agents confirmed to be effective against the tumor in vitro. Further intensive follow-up study is necessary for this infant.

\section{References}

1) Ajani JA, Baker FL, Spitzer G, Kelly A, Brock W, Tomasovic B, Singletary SE, McMurtrey M, Plager $\mathrm{C}$ : Comparison between clinical response and in vitro drug sensitivity of primary human tumors in the adhesive tumor cell culture system. J Clin Oncol 5: 1912 1921,1987

2) Allen JC, Wisoff J, Pierce J, Helson L: Choroid plexus carcinoma: Responses to chemotherapy alone in newly diagnosed young children. Ann Neurol 26: 461,1989

3) Alley MC, Scudiero DA, Monks A, Hursey ML, Czerwinski MJ, Fine DL, Abbott BJ, Mayo JG, Shoemaker RH, Boyd MR: Feasibility of drug screening with panels of human tumor cell lines using a microculture tetrazolium assay. Cancer Res 48: 589601,1988

4) Ausman JI, Shrontz C, Chason J, Knighton RS, Pak H, Patel S: Aggressive choroid plexus papilloma. Surg Neurol 22: 472-476, 1984

5) Boyd MC, Steinbok P: Choroid plexus tumors: Problems in diagnosis and management. J Neurosurg 66: 800-805, 1987

6) Carpenter DB, Michelsen WJ, Hays AP: Carcinoma of the choroid plexus. $J$ Neurosurg 56: 722-727, 1982

7) Dohrmann GJ, Collias JC: Choroid plexus carcinoma. J Neurosurg 43: 225-232, 1975

8) Duffner PK, Cohen ME, Horowitz M, Kun L, Burger PC, Kadota R, Valdez L, Phillips P, Krischer J: The treatment of choroid plexus carcinoma in infancy with chemotherapy. Ann Neurol 26: 460, 1989

9) Ellenbogen RG, Winston KR, Kupsky WJ: Tumors of the choroid plexus in children. Neurosurgery 25: 327-335, 1989

10) Gianella-Borradori A, Zeltzer PM, Bodey B, Nelson $\mathbf{M}$, Britton $\mathbf{H}$, Marlin A: Choroid plexus tumors in childhood. Cancer 69: 809-816, 1992

11) Haddad SF, Menezes AH, Bell WE, Godersky JC, Afifi AK, Bale JF: Brain tumors occurring before 1 year of age: A retrospective review of 22 cases in an 11-year period (1977-1987). Neurosurgery 29: 8-13, 1991

12) Lewis P: Carcinoma of the choroid plexus. Brain 90: 177-186, 1967 
13) Matsuda M, Uzura S, Nakasu S, Handa J: Primary carcinoma of the choroid plexus in the lateral ventricle. Surg Neurol 36: 294-299, 1991

14) Matsutani H, Nakamura $O$, Nagashima $T$, Asai A, Fujimaki $T$, Tanaka $H$, Nakamura $M$, Ueki $K$, Tanaka $Y$, Matsuda $T$ : Intra-operative radiation therapy for malignant brain tumours: Rationale, method, and treatment results of cerebral glioblastomas. Acta Neurochir (Wien) 131: 80-90, 1994

15) McGirr SJ, Ebersold MJ, Scheithauer BW, Quast LM, Shaw EG: Choroid plexus papillomas: Longterm follow-up results in a surgically treated series. $J$ Neurosurg 69: 843-849, 1988

16) Nelson RL: The comparative clinical pharmacology and pharmacokinetics of vindesine, vincristine, and vinblastine in human patients with cancer. Med Pediatr Oncol 10: 115-127, 1982

17) Packer RJ, Perilongo G, Johnson D, Sutton LN, Vezina G, Zimmerman RA, Ryan J, Reaman G, Schut L: Choroid plexus carcinoma of childhood. Cancer 69: 580-585, 1992

18) Pendergrass TW, Milstein JM, Geyer JR, Mulne AF, Kosnik EJ, Morris JD, Heideman RL, Ruymann FB, Stuntz JT, Bleyer WA: Eight drugs in one day chemotherapy for brain tumors: Experience in 107 children and rationale for preparation chemotherapy. $J$ Clin Oncol 5: 1221-1231, 1987

19) Russel DS, Rubinstein LJ: Pathology of Tumours of the Nervous System, ed 5. Baltimore, Williams \& Wilkins, 1989, pp 394-403
20) Sakai N, Yamada $H$, Andoh $T$, Hirata $T$, Nishimura Y, Miwa Y, Shimizu K, Yanagawa S: Intraoperative radiation therapy for malignant glioma. Neurol Med Chir (Tokyo) 31: 702-707, 1991

21) Sakai $N$, Yamada $H$, Andoh $T$, Takada $M$, Hirata $T$, Funakoshi $T$, Doi $H$, Yanagawa $S$ : Intraoperative radiation therapy for malignant glioma. Neurol Med Chir (Tokyo) 29: 312-318, 1989 (in Japanese)

22) Tomita T, McLone DG, Naidich TP: Mural tumors with cysts in the cerebral hemispheres of children. Neurosurgery 19: 998-1005, 1986

23) Tomita $T$, Naidich TP: Successful resection of choroid plexus papillomas diagnosed at birth: Report of two cases. Neurosurgery 20: 774-779, 1987

24) Vinken PJ, Slooff ACJ: A case of carcinoma of the choroid plexus in a child. Zentralbl Neurochir 26: 313-317, 1965

25) Zulch KJ: Brain Tumors. Their Biology and Pathology, ed 3. Berlin, Springer, 1986, pp 276-283

26) Zurek WZ, Ojima $Y$, Anderson LL, Collins GJ, Oberfield RA, Sullivan RD: Pharmacological studies of methotrexate in man. Surg Gynec Obstet 126: 331338,1968

Address reprint requests to: T. Kumabe, M.D., Department of Neurosurgery, Tohoku University School of Medicine, 1-1 Seiryo-machi, Aoba-ku, Sendai 98077, Japan. 